\title{
PAPER
}

\section{Occipital hypoperfusion in Parkinson's disease without dementia: correlation to impaired cortical visual processing}

\author{
Y Abe, T Kachi, T Kato, Y Arahata, T Yamada, Y Washimi, K Iwai, K Ito, N Yanagisawa, \\ G Sobue
}

See end of article for authors' affiliations

......................

Correspondence to: Dr Y Abe, Department of Neurology, Chubu

National Hospital, Obu, Aichi 474-8511, Japan; yujiabe@chubu-nh.go.jp

Received 5 August 2002 Accepted in revised form 22 October 2002

\begin{abstract}
Objective: The purpose of this study was to analyse changes in regional cerebral blood flow (rCBF) in Parkinson's disease (PD) without dementia.

Methods: Twenty eight non-demented patients with PD and 17 age matched normal subjects underwent single photon emission computed tomography with $\mathrm{N}$-isopropyl-p-[ $\left.{ }^{123} \mid\right]$ iodoamphetamine to measure rCBF. The statistical parametric mapping 96 programme was used for statistical analysis. Results: The PD patients showed significantly reduced rCBF in the bilateral occipital and posterior parietal cortices $(p<0.01$, corrected for multiple comparison $p<0.05)$, when compared with the control subjects. There was a strong positive correlation between the score of Raven's coloured progressive matrices (RCPM) and the $\mathrm{rCBF}$ in the right visual association area $(p<0.01$, corrected for multiple comparison $\mathrm{p}<0.05$ ) among the PD patients.

Conclusions: This study showed occipital and posterior parietal hypoperfusion in PD patients without dementia. Furthermore, it was demonstrated that occipital hypoperfusion is likely to underlie impairment of visual cognition according to the RCPM test, which is not related to motor impairment.
\end{abstract}

k t has been reported in previous studies that patients with Parkinson's disease (PD), even those without dementia, showed changes in regional cerebral blood flow $(\mathrm{rCBF}) .^{1-8}$ However, the findings of these investigations have been inconsistent. Frontal $^{12}$ parietal $^{3}$, , temporal, ${ }^{4}$ or global ${ }^{15}$ cortical hypoperfusion, or unchanged blood flow ${ }^{6-8}$ have been reported. This inconsistency is considered to be attributable, not only to the heterogeneity among PD patients, but also to the lack of standardisation of image-analysing methods. In most previous studies, visually placed region of interest (ROI) analysis methods were used to evaluate the alterations of rCBF. This approach is limited in that the manual placement of ROI gives rise to the observer biases and large areas of the brain are left unexplored.

Statistical parametric mapping (SPM), developed by Frinston et al, is a voxel based statistical technique that is used to examine regional changes in imaging data. ${ }^{910}$ This is entirely automated and objective, and can completely overcome the disadvantage of earlier ROI analysis methods. Recently, the SPM programme has been widely used to examine regional dysfunction of the brain in various neurological diseases. ${ }^{511-13}$

The purpose of this study was to analyse the rCBF in PD patients without dementia on a voxel by voxel basis using single photon emission computed tomography (SPECT) with $\mathrm{N}$-isopropyl-p-[ $\left.{ }^{[23} \mathrm{I}\right]$ iodoamphetamine $\left({ }^{123} \mathrm{I}\right.$-IMP) and the SPM programme. We compared the rCBF in PD patients to that in age matched normal subjects. In addition, we investigated the relation between the $\mathrm{rCBF}$ and clinical features in PD patients.

\begin{tabular}{lll} 
Table 1 & \multicolumn{2}{l}{ Clinical features of subjects } \\
\hline & $\begin{array}{l}\text { Normal controls } \\
(\mathrm{n}=17)\end{array}$ & $\begin{array}{l}\text { Patients with Parkinson's } \\
\text { disease }(\mathrm{n}=28)\end{array}$ \\
\hline Age $(\mathrm{y}){ }^{*}$ & $69.6(10.2)$ & $67.3(7.3)$ \\
Sex $(\mathrm{F} / \mathrm{M})$ & $9 / 8$ & $17 / 11$ \\
Duration of iluness $(\mathrm{y})^{*}$ & $8.6(4.6)$ \\
UPDRS motor score* & & $35.2(12.9)$ \\
\hline *Mean (SD). &
\end{tabular}

\section{METHODS \\ Subjects}

Twenty eight PD patients without dementia and 17 age matched normal control subjects were included in this study (table 1). The 28 PD patients were diagnosed with the United Kingdom Parkinson's Disease Society Brain Bank criteria for clinical diagnosis of idiopathic PD, ${ }^{14}$ and the extrapyramidal symptoms were scored according to the motor examination score of the Unified Parkinson's Disease Rating Scale (UPDRS). ${ }^{15}$ Patients who had visual hallucination were excluded. No abnormal intensity or obvious cortical atrophy was seen on magnetic resonance imaging of the brain of any patient. None of the patients had any other illnesses or were taking any medication, except for antiparkinsonian drugs. All 28 patients had been taking levodopa treatment at the time of SPECT scanning. In addition, dopamine receptor agonists had been used in 12 patients, six patients had been treated with low dose anticholinergic agents, droxydopa had been given to two patients, and three patients had been taking amantadine hydrochloride.

None of the 17 normal control subjects had a history of any neurological or psychiatric disorders, and the neurological examination of each control subject was normal.

All of the PD and control subjects were assessed using the mini-mental state examination (MMSE), ${ }^{16}$ and 25 PD patients and 14 control subjects also took the Raven's coloured progressive matrices (RCPM). ${ }^{17}$ For both SPECT scanning and cognitive test, all patients were examined after overnight withdrawal of medication.

Informed consent was obtained from every subject before the study. Permission to perform this study was obtained from the ethical committee of Chubu National Hospital.

\footnotetext{
Abbreviations: PD, Parkinson's disease; $\mathrm{rCBF}$, regional cerebral blood flow; RCPM, Raven's coloured progressive matrices; MMSE, mini-mental state examination; ROI, region of interest; SPM, statistical parametric mapping
} 


\section{Image acquisition}

${ }^{123}$ I-IMP (Nihon Mediphysics, Hyogo, Japan), 222 MBq (6 $\mathrm{mCi})$, was injected into an antecubital vein while the subjects laid in a supine position with eyes closed in a quiet room. A single blood sample was obtained from the brachial artery between 9 and 10 minutes after the ${ }^{123}$ I-IMP administration. SPECT scanning was carried out between 15 and 45 minutes after injection using a two head rotating GCA 7200DI gammacamera (Toshiba, Tokyo, Japan) fitted with low energy, high resolution collimators. The data were acquired in a $128 \times 128$ matrix through a $180^{\circ}$ rotation at an angle interval of $4^{\circ}$. The projection data were prefiltered through a Butterworth filter, and then reconstructed using a Ramp backprojection filter. Chang's attenuation correction ${ }^{18}$ and scattering correction using the triple energy window method ${ }^{19}$ were applied to the reconstructed images. The in-plane spatial resolution was $11.1 \mathrm{~mm}$ in full width at half maximum (FWHM). The final image slices were set up parallel to the orbitomeatal line and were obtained at an interval of $3.44 \mathrm{~mm}$ through the entire brain. The rCBF images were quantitated according to the IMP-ARG method..$^{20}$ This method is based on the two compartment model for tracer kinetics, and uses a standard arterial input calibrated by the radioactivity of a single arterial whole blood sample, a standard lipophilic fraction of ${ }^{123} \mathrm{I}$-IMP in whole blood and fixed distribution volume of ${ }^{123}$ I-IMP. All images were transferred to a Sun workstation (Sun Microsystems, Mountain View, CA, USA) for further analysis.

\section{Data analysis}

The data were analysed using the statistical parametric mapping 96 (SPM96; Wellcome Department of Cognitive Neurology, Institute of Neurology, London, UK) ${ }^{9}{ }^{10}$ implemented in MATLAB (Math Works, Sherborn, MA, USA). Each image was transformed into the standard anatomical space with the programme provided by the Montreal Neurological Institute. ${ }^{21}$ All of the images resulting from the normalisation procedure were visually acceptable. The global CBF of PD patients was

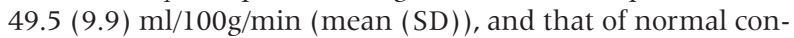
trols was $52.4(13.1) \mathrm{ml} / \mathrm{l00g} / \mathrm{min}$. There was no significant difference of the mean global CBF in the two groups, but the apparent interindividual variation of the global CBF was seen in each group. Therefore, in the following analyses, proportional scaling was applied to adjust the mean whole brain activity to $50 \mathrm{ml} / 100 \mathrm{~g} / \mathrm{min}$ to avoid interindividual variation in global CBF. The grey matter threshold was 0.8 .

For comparison between the PD group and the control group, the normalised images of the non-demented patients with PD and those of the normal subjects were compared by a voxel by voxel $t$ statistics. The resulting statistical parametric maps of $t$ statistics, $\operatorname{SPM}\{\mathrm{t}\}$, were transformed to maps of the unit normal distribution, $\operatorname{SPM}\{z\}$. The statistical significance was chosen at a level of $\mathrm{z}>2.33$ (equivalent to an uncorrected $\mathrm{p}<0.01)$. To correct for multiple comparisons, the significance of the difference between each detected brain region was estimated using distributional approximations from theory of Gaussian field, in terms of spatial extent and peak height. A corrected p value of 0.05 was used as the final threshold for significance.

Next, we compared the images of the PD patients to examine whether there were any voxels in which the rCBF was significantly correlated with various clinical characteristics including the duration of illness, the UPDRS motor score, the MMSE score, and the RCPM score. Each value of the clinical characteristics was used as a covariate of interest, and both the values of the global rCBF and age were used as confounding covariates. The statistical significance was chosen at a level of $\mathrm{z}>2.33$ (equivalent to an uncorrected $\mathrm{p}<0.01$ ). A corrected $\mathrm{p}$ value of 0.05 was chosen in multiple comparisons.
Table 2 Brain regions in which the rCBF of the PD group was significantly lower than that of the normal control group (SPM analysis)

\begin{tabular}{|c|c|c|c|c|c|}
\hline Cerebral region & $\begin{array}{l}\text { Brodmann's } \\
\text { area }\end{array}$ & $x$ & y & z & Z \\
\hline L middle occipital gyrus & $18,19,39$ & -34 & -78 & 8 & 4.63 \\
\hline R middle occipital gyrus & $18,19,39$ & 28 & -84 & 14 & 4.28 \\
\hline $\mathrm{L}$ angular gyrus & 39 & -46 & -54 & 34 & 3.43 \\
\hline $\mathrm{R}$ angular gyrus & 39 & 44 & -56 & 38 & 3.98 \\
\hline $\mathrm{L}$ cuneus & 18 & -26 & -86 & 0 & 3.37 \\
\hline$R$ cuneus & 18 & 8 & -88 & 14 & 2.57 \\
\hline L calcarine sulcus & 17 & -18 & -94 & -8 & 3.69 \\
\hline L precuneus & 7 & -10 & -70 & 42 & 3.37 \\
\hline $\mathrm{L}$ lingual gyrus & 18 & -18 & -84 & -16 & 3.36 \\
\hline L superior occipital gyrus & 19 & -32 & -84 & 30 & 3.34 \\
\hline L superior parietal lobule & 7 & -12 & -72 & 52 & 3.23 \\
\hline
\end{tabular}

$x, y, z=$ coodinates of the peak in the standard anatomical space; $\mathrm{Z}=\mathrm{Z}$ score of maximal peak; $\mathrm{L}=$ left; $\mathrm{R}=$ right. $\mathrm{p}<0.01$, corrected for multiple comparisons.

\section{RESULTS}

\section{Clinical manifestations}

The total UPDRS motor examination score ranged from 3 to 56 , and the mean (SD) score was 34.8 (13.2). The mean MMSE score of the $28 \mathrm{PD}$ patients was 28.1 (2.1) (range, 25 to 30 ), and that of the 17 control subjects was 28.5 (2.0) (range, 25 to 30). The MMSE score of the PD group did not differ significantly from that of the control group. The mean RCPM score was 24.4 (5.2) (range, 16 to 33) for the 25 PD patients, and was 28.2 (3.5) (range, 25 to 34 ) for the 10 control subjects. The RCPM score of the PD group was significantly lower than that of the control group $(\mathrm{p}<0.05)$.

There was no correlation between the age, the duration of illness, the UPDRS motor examination score, the MMSE score, and the RCPM score in the PD group.

\section{Comparison of the rCBF between the PD and normal control groups}

Twenty eight PD patients without dementia and 17 control subjects underwent SPECT scanning, and the images of the PD and control groups were compared. According to SPM analysis, the rCBF in the bilateral occipital cortices and the bilateral posterior parietal cortices in the PD group were significantly lower than those in the respective area in the control group $(p<0.01$, corrected $p<0.05)$ (table 2, fig 1 ).
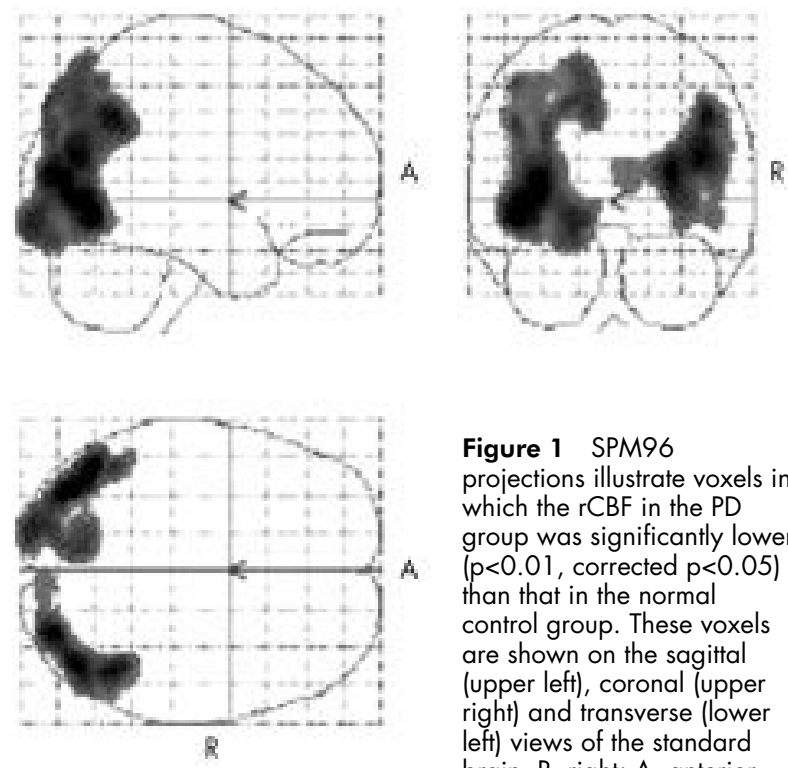

Figure 1 SPM96

projections illustrate voxels in which the $\mathrm{rCBF}$ in the PD group was significantly lower $(p<0.01$, corrected $p<0.05)$ than that in the normal control group. These voxels are shown on the sagittal (upper left), coronal (upper right) and transverse (lower left) views of the standard brain. $R$, right; $A$, anterior. 
Table 3 Brain regions in which there was a positive correlation between the RCPM score and the rCBF among 25 PD patients (SPM analysis)

\begin{tabular}{llllll}
\hline Cerebral region & $\begin{array}{l}\text { Brodmann's } \\
\text { area }\end{array}$ & $x$ & $y$ & $z$ & $z$ \\
\hline$R$ angular gyrus & 39 & 46 & -66 & 24 & 4.36 \\
R middle occipital gyrus & 19 & 40 & -70 & 18 & 4.34 \\
R inferior parietal lobule & 40 & 42 & -50 & 46 & 3.09 \\
\hline
\end{tabular}

$x, y, z=$ coodinates of the peak in the standard anatomical space; $Z=Z$ score of maximal peak; $R=$ right. $p<0.01$, corrected for multiple comparisons.

There was no brain region in which the rCBF was significantly higher in the PD group than in the control group.

\section{Correlation between clinical characteristics and $\mathrm{rCBF}$ in the PD patients without dementia}

There was a positive correlation between the RCPM score and the $\mathrm{rCBF}$ in the right dorsolateral occipital and the right posterior parietal cortices $(\mathrm{p}<0.01$, corrected $\mathrm{p}<0.05)$ among 25 PD patients (table 3, fig 2). In most of these regions, the rCBF was reduced significantly in the PD group compared with the normal control group. There was no correlation between the duration of illness, the UPDRS motor examination score, or the MMSE score and the value of $\mathrm{rCBF}$ for any brain region.

\section{DISCUSSION}

This study showed that the rCBF in the non-demented PD patients was significantly lower than that in the age matched normal subjects in the bilateral occipital and posterior parietal cortices. It is well known that patients with PD, even those who do not have dementia, often develop various kinds of cognitive abnormalities that are closely related to visual dysfunction. Loss of luminance and colour contrast sensitivity and the impairment in preattentive cortical visual processing have been reported..$^{22-24}$ Furthermore, neuropsychological studies have revealed that PD patients have visuospatial deficit. ${ }^{25}{ }^{26}$ It seems appropriate to conclude that occipital dysfunction is a common feature of PD patients without dementia.

Actually, in our study, the mean RCPM score in the PD group was significantly lower than that in the normal control group, although the MMSE score of the PD group and that of the control group did not differ. RCPM is used for evaluation of visual perception, especially visuospatial attention, as the person taking the test must visually analyse form, colour, and linear slope ${ }^{27}$ Moreover, RCPM is one of the most appropriate batteries to test visual perceptual function purely, because it requires very little motor response. Therefore, in PD patients, the RCPM score is not likely to be affected by the poor motor ability. In fact, the RCPM score did not correlate to the UPDRS motor score in our PD patients. The impairment in RCPM test is clearly distinct from dementia, and is not considered to be secondary to the motor dysfunction.

In this study, we found that there was a strong positive correlation between the RCPM score and the rCBF in the right dorsolateral occipital area, corresponding to the visual association cortex, and the right posterior parietal area among nondemented PD patients. In these areas, there was no correlation between the UPDRS motor score and the $\mathrm{rCBF}$, therefore, it may be reasonable to suppose that the reduction of rCBF in the visual association area purely reflects the impairment in cortical visual processing. Although some previous studies demonstrated occipital hypoperfusion and glucose hypometabolism, ${ }^{58-31}$ there has been no previous study showing that the correlation between occipital hypometabolism or hypoperfusion and clinical abnormality related with visual
A
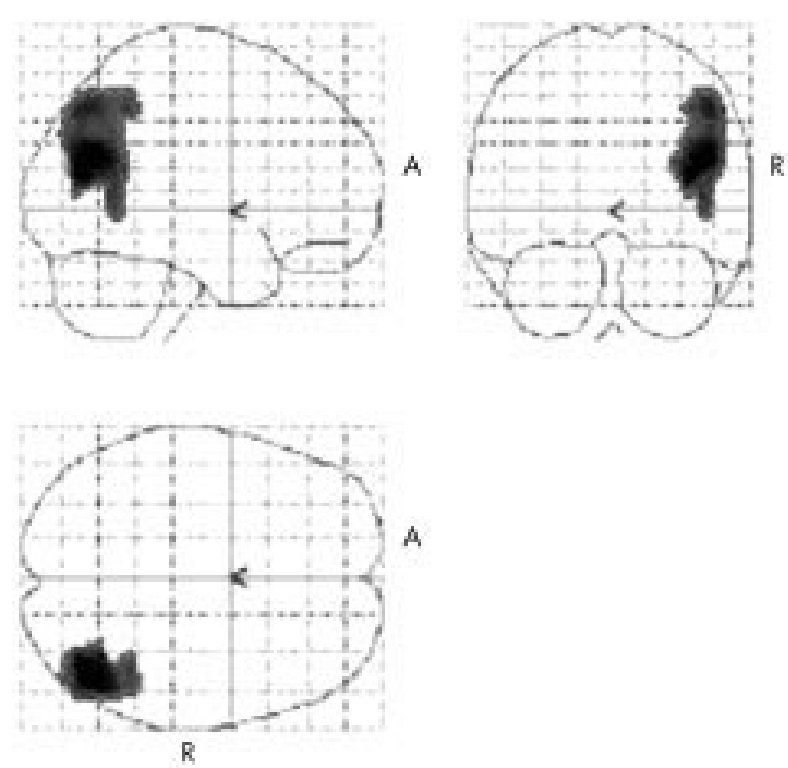

B

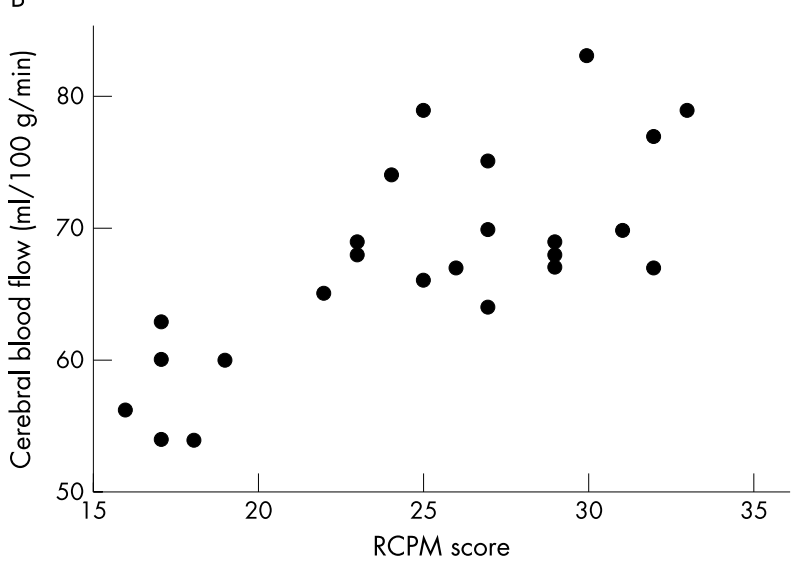

Figure 2 (A) SPM96 projections illustrate voxels in which there is a positive correlation $(p<0.01$, corrected $p<0.05)$ between the RCPM score and the $\mathrm{rCBF}$ among 25 PD patients. $\mathrm{R}$, right; $\mathrm{A}$, anterior. (B) Scatter plot between the RCPM score and the rCBF at the voxel in the right middle occipital gyrus where there is a positive correlation $(p<0.005, r=0.73$, Spearman's correlation coefficient by ranks) between the RCPM score and the rCBF. *The mean whole brain activity was adjusted to $50 \mathrm{ml} / 100 \mathrm{~g} / \mathrm{min}$.

dysfunction was directly proved. This is the first study to demonstrate this correlation. The reason why the correlation was seen only in the right hemisphere is not clear, but this is possibly associated with the dominance of the right hemisphere in visuospatial attention. ${ }^{32}{ }^{33}$

The underlying mechanism of the reduction of $\mathrm{rCBF}$ in the occipital lobe of PD patients remains unknown. In the previous report, a reduction of amplitude in the pattern electroretinogram ${ }^{34}$ was demonstrated in PD patients. This abnormality has been considered to be attributable to diminished dopaminergic neurons in the retina, and this retinal dysfunction may be responsible for the occipital hypoperfusion. Furthermore, PD patients may have primary pathological findings in the cortices. However, morphological studies have not disclosed primary abnormalities, such as neuronal loss, gliosis, and appearance of Lewy bodies, in these brain regions in non-demented PD patients. ${ }^{35}{ }^{36}$ Another explanation, which seems much more plausible, is a functional deficit of the cortex that results from damage to the 
subcortical structure. There is considerable evidence supporting the presence of a corticostriatal projection which arises from the entire cortical region and projects to the striatum. ${ }^{37} 38$ The compact zone of the substantia nigra also gives off efferents to the striatum, and is widely believed to modify the activity of the cortical output to the striatum. ${ }^{37}{ }^{38}$ Therefore, the loss of nigrostriatal neurons may impair the association between the cortical region and the striatum, and consequently may reduce cortical activity. The research group of DennyBrown and Yanagisawa reported the intimate functional connection between the visual association areas and the striatum..$^{39}$ They demonstrated in monkeys that extensive destruction of the posterior part of the putamen was followed by a remarkable loss of visual attention and inhibition of grasp and traction response, and that these signs also resulted from destruction of the visual association cortex and the posterior parietal cortex. The physical connection of the visual association cortex and the posterior parietal cortex with the posterior part of the putamen was also described by Kemp and Powell. ${ }^{41}$

It is difficult to detect the correlation between RCPM score and the $\mathrm{rCBF}$ in the dorsolateral visual association areas, if conventional ROI analysis methods had been used. Because the dorsolateral visual association area is located around the parieto-occipital junction, most investigators avoid setting of ROIs in this area. On the other hand, using the SPM program, whole brain lesions can be explored by a voxel based statistical technique. It has become possible using the SPM program to detect that occipital hypoperfusion is related to the visual dysfunction according to the RCPM test.

In conclusion, we confirmed that occipital hypoperfusion is a common feature of PD patients without dementia. It was demonstrated that occipital hypoperfusion is likely to reflect the visual impairment examined with the RCPM test that is not related to the motor impairment.

\section{Authors' affiliations}

Y Abe, K Iwai, G Sobue, Department of Neurology, Nagoya University School of Medicine, Nagoya, Japan

Y Abe, T Kachi, Y Arahata, T Yamada, Y Washimi, K Iwai, Department of Neurology, Chubu National Hospital, Aichi, Japan

T Kato, K Ito, Department of Biofunctional Research, National Institute for Longevity Sciences, Aichi, Japan

N Yanagisawa, Department of Neurology, Kanto Rosai Hospital, Kanagawa, Japan

Funding: this research was partly supported by a Health Sciences Research Grant for Comprehensive Research on Aging and Health and a Health Sciences Research Grant for Research on Brain Science from the Ministry of Health, Labour and Welfare of Japan.

Competing interests: none declared.

\section{REFERENCES}

1 Wolfson LI, Leenders KL, Brown LL, et al. Alterations of regional cerebral blood flow and oxygen metabolism in Parkinson's disease. Neurology 1985:35:1399-405

2 Defebvre L, Lecouffe $P$, Destee A, et al. Tomographic measurements of regional cerebral blood flow in progressive supranuclear palsy and Parkinson's disease. Acta Neurol Scand 1995;92:235-41.

3 Tachibana $\mathbf{H}$, Kawabata K, Tomino $Y$, et al. Brain perfusion imaging in Parkinson's disease and Alzheimer's disease demonstrated by three-dimensional surface display with 1231-iodoamphetamine. Dementia 1993;4:334-41

4 Jagust WJ, Reed BR, Martin EM, et al. Cognitive function and regional cerebral blood flow in Parkinson's disease. Brain 1992;115:521-37.

5 Imon Y, Matsuda H, Ogawa M, et al. SPECT image analysis using statistical parametric mapping in patients with Parkinson's disease. J Nucl Med 1999;40:1583-9.

6 Perlmutter JS, Raichle ME. Regional blood flow in hemiparkinsonism. Neurology 1985;35:1127-34.

7 Spampinato U, Habert MO, Mas JL, et al. (99mTC)-HM-PAO SPECT and cognitive impairment in Parkinson's disease: a comparison with dementia of the Alzheimer type. J Neurol Neurosurg Psychiatry 1991;54:787-92.

8 Wang SJ, Liu RS, Liu HC, et al. Technetium-99m hexamethylpropylene amine oxime single photon emission tomography of the brain in early Parkinson's disease: correlation with dementia and lateralization. Eur J Nucl Med 1993;20:339-44.
9 Friston KJ, Ashburner J, Frith CD et al. Spatial registration and normalization of images. Hum Brain Mapp 1995;3:165-89.

10 Friston KJ, Holmes AP, Worsley KJ, et al. Statistical parametric maps in functional imaging: a general linear approach. Hum Brain Mapp 1995:2:189-210

11 Roelcke U, Kappos L, Lechner-Scott J, et al. Reduced glucose metabolism in the frontal cortex and basal ganglia of multiple sclerosis patients with fatigue: a ${ }^{18} \mathrm{~F}$-fluorodeoxyglucose positron emission tomography study. Neurology 1997;48:1566-71.

12 Desgranges B, Baron JC, de la Sayette V, et al. The neural substrates of memory systems impairment in Alzheimer's disease. A PET study of resting brain glucose utilization. Brain 1998;121:611-31.

13 Van Bogaert P, David P, Gillain CA, et al. Perisylvian dysgenesis. Clinical, EEG, MRI and glucose metabolism features in 10 patients. Brain 1998;121:2229-38.

14 Hughes AJ, Daniel SE, Kilford L, et al. Accuracy of clinical diagnosis of idiopathic Parkinson's disease: a clinico-pathological study of 100 cases. J Neurol Neurosurg Psychiatry 1992;55:181-4.

15 Fahn S, Elton RL, Members of the UPDRS Development Committee. Unified Parkinson's disease rating scale. In: Fahn S, Marsden CD, Calne DB, et al, eds. Recent development in Parkinson's disease. Florham Park NJ: MacMillan Healthcare Information, 1987:153-63, 293-304.

16 Folstein MF, Folstein SE, McHugh PR. "Mini-mental state". A practical method for grading the cognitive state of patients for the clinician. $J$ Psychiatr Res 1975;12:189-98.

17 Raven J. Guide to using the coloured progressive matrices. London: Lewis, 1965.

18 Chang LT. A method for attenuation correction in radionuclide computed tomography. IEEE Trans Nucl Sci 1978;25:638-43.

19 Ogawa K. A practical method for position-dependent compton-scatter correction in single photon emission CT. IEEE Trans Med Imaging 1991;10:408-12.

20 lida $\mathbf{H}$, Itoh $\mathrm{H}$, Nakazawa $M$, et al. Quantitative mapping of regional cerebral blood flow using iodine-123-IMP and SPECT. J Nucl Med 1994;35:2019-30.

21 Evans AC, Collins DL, Milner B. An MRI-based stereotactic atlas from 250 young normal subjects. Soc Neurosci Abstr 1992;18:408.

22 Bodis-Wollner I, Marx MS, Mitra S, et al. Visual dysfunction in Parkinson's disease. Loss in spatiotemporal contrast sensitivity. Brain 1987; 110:1675-98.

23 Haug BA, Kolle RU, Trenkwalder C, et al. Predominant affection of the blue cone pathway in Parkinson's disease. Brain 1995;1 18:771-8.

24 Lieb K, Brucker S, Bach M, et al. Impairment in preattentive visual processing in patients with Parkinson's disease. Brain 1999;122:30313.

25 Pirozzolo FJ, Hansch EC, Mortimer JA, et al. Dementia in Parkinson disease: a neuropsychological analysis. Brain Cogn 1982;1:71-83.

26 Boller F, Passafiume D, Keefe NC, et al. Visuospatial impairment in Parkinson's disease. Role of perceptual and motor factors. Arch Neurol 1984;41:485-90.

27 Tanaka F, Kachi T, Yamada T, et al. Auditory and visual event-related potentials and flash visual evoked potentials in Alzheimer's disease: correlations with Mini-Mental State Examination and Raven's coloured progressive matrices. J Neurol Sci 1998;156:83-8.

28 Peppard RF, Martin WR, Carr GD, et al. Cerebral glucose metabolism in Parkinson's disease with and without dementia. Arch Neurol 1992;49:1262-8.

29 Eberling JL, Richardson BC, Reed BR, et al. Cortical glucose metabolism in Parkinson's disease without dementia. Neurobiol Aging 1994;15:329-35

30 Bohnen NI, Minoshima S, Giordani B, et al. Motor correlates of occipital glucose hypometabolism in Parkinson's disease without dementia. Neurology 1999;52:541-6.

31 Hu MT, Taylor-Robinson SD, Chaudhuri KR, et al. Cortical dysfunction in non-demented Parkinson's disease patients: a combined ${ }^{31}$ P-MRS and ${ }^{18}$ FDG-PET study. Brain 2000;1 23:340-52.

32 Warrington EK, James $M$, Kinsbourne $M$. Drawing disability in relation to laterality of cerebral lesion. Brain 1966;89:53-82

33 Benson DF, Barton MI. Disturbances in constructional ability. Cortex 1970:6:19-46.

34 Ikeda H, Head GM, Ellis CJ. Electrophysiological signs of retinal dopamine deficiency in recently diagnosed Parkinson's disease and a follow up study. Vision Res 1994;34:2629-38.

35 Jellinger KA. Pathology of Parkinson's disease. Changes other than the nigrostriatal pathway. Mol Chem Neuropathol 1991;14:153-97.

36 Hughes AJ, Daniel SE, Blankson S, et al. A clinicopathologic study of 100 cases of Parkinson's disease. Arch Neurol 1993;50:140-8.

37 Alexander GE, Crutcher MD. Functional architecture of basal ganglia circuits: neural substrates of parallel processing. Trends Neurosci 1990; 13:266-71

38 Parent A, Hazrati LN. Functional anatomy of the basal ganglia. I. The cortico-basal ganglia-thalamo-cortical loop. Brain Res Brain Res Rev 1995;20:91-127.

39 Denny-Brown D, Yanagisawa N, Kirk E. The localization of hemispheric mechanism of visually directed reaching and grasping. In: Zülch KJ, Kreutzfeldt O, Galbraith GC, eds. Cerebral localization. Berlin: Springer, 1975:62-75.

40 Denny-Brown D, Yanagisawa N. The role of the basal ganglia in initiation of movement. In: Yahr $M$, ed. The basal ganglia. New York: Raven Press, 1976:115-49.

41 Kemp JM, Powell TP. The cortico-striate projection in the monkey. Brain 1970;93:525-46. 